\title{
Mini - review on the psychiatric comorbidities in epilepsy - possible relevance for some metabolic changes?
}

\author{
Raluca Elena Duță, Cristina Elena Nedelcu, \\ Alin Ciobîcă, Daniel Timofte
}

Raluca Elena Duță - master student, Department of Research, Faculty of Biology, Alexandru Ioan Cuza University, B dul Carol I, no 11, Iasi, Romania

Cristina Elena Nedelcu - clinician psycholog, psychotherapist, Socola Institute of Psychiatry Iaşi, Romania

Alin Ciobîcă - researcher, Department of Research, Faculty of Biology, Alexandru Ioan Cuza University, B dul Carol I, no 11, Iasi, Romania Academy of Romanian Scientists, Splaiul Independentei nr. 54, sector 5, 050094 București, Romania,Center of Biomedical Research, Romanian Academy, Iași, B-dul Carol I, no 8, Romania

Daniel Timofte - MD, PhD, professor, "Grigore T.Popa" University of Medicine and Pharmacy, 16, Universitatii Street, 700115, Iasi, Romania

\begin{abstract}
Epilepsy is one of the most important neurological disorders nowadays, with a variety of studies describing its mechanisms and complex pathophysiology. However, lately there is also an increased interest in understanding the psychiatric comorbities associated with the epileptic pathology. Thus, we will describe here aspects such as the importance of anxiety, depression, personality disorders, the suicidal ideation in epilepsy, as well as the correlations that might exist between the specific treatment for epilepsy and its psychiatric-related effects. In conclusion, it seems that the associated psychiatric manifestations from epilepsy have an increased important in the pathophysiology of this disorder and deserve further investigation.
\end{abstract}

\section{KEYWORDS:}

Psychiatric comorbidities, epilepsy, metabolic

\section{INTRODUCTION}

Epilepsy is one of the most common and disabling (1) serious neurological disorders and affects approximately 50 million people of all ages worldwide, sex and socioeconomic status. Among these individuals, about $80 \%$ of them live in countries that have small or medium gross domestic product. Most often 


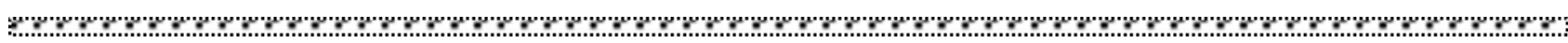
people suffering from this disease and their families have frequent stigma and discrimination problems. EEG-fMRI tests have shown that cognitive networks can be coactivated during epileptiform discharges, thus altering their function and connectivity ovr time $(2,3,4)$.

An epileptic seizure is a transient onset where there is no abnormal signs and/or abnormal symptoms of excessive or synchronous neuronal activity in the brain (5). Crises can be divided into several types and depending on the region: focal - with the origin in the limited network of a sphere of the brain, or they can be generalized by their appearance within and rapidly involving these networks in the two hemispheres $(6,7)$.

Hermann and his colleagues argued in 1986 that the variables that contribute to the psycho-pathological development are divided into three main groups: the clinical factors associated with the natural history of epilepsy, the age at which the disease began, the duration of the seizure and its type, the etiology and seizure control. Medication factors including AED, dose and quantity of active substance, respectively psychosocial factors including astigmatism, perceived discrimination, social and socio-economic support are also important in this context (6). Psychiatric comorbidity has begun to be increasingly studied, although its heuristic value has long been recognized. There is no definition to be used in a uniform manner, comorbidity can be defined as the presence of one or more additional conditions that appear together with another condition that is primary (8). The full exploitation of the mechanisms requires an interdisciplinary approach to the investigation of the topology, the evaluation and the underlying comorbidity methods, as well as certain clinical and epidemiological experimental studies. This more precise specification of the patterns could help to identify the usual biochemical, cognitive markers relevant in the etiology of specific mental disorders, residing in comorbid conditions (9). Epilepsy is a phenomenon as a model in the history of neurology intersected with psychiatry, studying the cognitive, affective interests and behaviour of patients (10).

A model of progression of the symptoms of comorbidity associated with panic disorders is realized from the precursor conditions such as: biological or cognitive vulnerability, anxiety syndrome, situational risk factors in the patient's daily life, followed by the 6 phases: the onset of spontaneous seizures. Panic, and sensibilities disorders, panic, and syndrome agoraphobia, depress major demoralization last phase being dependent behaviour. For most people who are epileptic or their families, the thoughts of this disease, but also what the seizures represent, are caused or can cause comorbid conditions, including behavioural disorders, depression or anxiety, which can lead to suicide (1).

The most common psychological comorbidities are depression and anxiety that are common in patients with epilepsy, being called comorbid disorders. 25-50 \% of epileptic cases present with depression, anxiety, psychotic disorders, cognitive and sonorous per modifications that occur in interictal, ictal or postictal states (8). In certain Neuroscience institutions (Epilepsy Canter of the Institute of Neuroscience, University of Cincinnati), all patients with epilepsy are routinely analyzed and observed if they have depression or anxiety, using the Neurological Depression Inventory for Epilepsy (NDDI-E) Generalized Anxiety 7 (GAD-7), being recommended to make this knowledge of behaviour both in children and adults. 
A good understanding between comorbidity and epilepsy is essential for more pronounced recognition and more effective treatment, but also for informing clinicians about the needs of epileptics. This can be defined by improving the care of these people with epilepsy, together with the observed associations they can help to understand the common mechanisms underlying the link between epilepsy and the specific forms of psychopathology (11). The reported prevalence of comorbid psychopathology in epilepsy varied greatly during the studies and reflected methodological limitations such as possible selection due to the recruitment of epilepsy cases from unrepresentative clinical populations or measurement due to the lack of diagnostic assessment tools to determine psychopathology $(12,13,14,15,16)$.

Apart from this problem being the link between comorbidity and epilepsy, there are also problems such as socio-economic disadvantages and other health problems of people with epilepsy $(17,18)$ that can lead to depression disorders, anxiety and depressive symptoms. Therefore, it is not known to what extent epilepsy may be associated with an increased risk of psychopathology, besides the effect of having a chronic medical or neurological condition (11).

\section{PSYCHIATRIC COMORBIDITIES IN EPILEPSY}

Psychiatric disorders are commonly seen in people with epilepsy and these can negatively influence the course of this condition and lead to an unfavourable response to treatment that contributes to poor quality of life and increased mortality $(17,18)$. However, psychopathology is often unrecognized and untreated in these persons and many questions remain about both the appearances and the nature of the relationships between epilepsy and specific psychiatric conditions (11).
One of the most complex studies on this matter was performed by Rai and his collaborators on psychiatric morbidity for adults that included 7403 detailed interviews with people in England. The epilepsy that was diagnosed by the physician was verified by self-report and extensive interviews were used for diagnosis and screening and this was how psychiatric disorders and neurodevelopmental were evaluated. About one third of the people interviewed with epilepsy met the criteria for ICD-10 diagnosis, which consists of anxiety or depressive disorder, compared with about 1 in 6 people who do not have epilepsy. Individuals with epilepsy at the interview had significant chances of having social phobias, agoraphobia, generalized anxiety disorder, depression, and suicide measures, these were stronger than in patients suffering from another disease (ex: asthma, diabetes). Epileptics have been diagnosed with a higher chance of having an autism spectrum disorder and an increased likelihood of being positive for eating disorders, as compared with people without epilepsy (11).

Psychiatric conditions in patients with epilepsy may also exhibit characteristics that are inconsistent with those commonly seen in different forms of the disorder. These comorbidities may have an increased risk of not being terminated or misleading in cohort studies. However, administrative and electronic health documents are an alternative to potential cohort studies, with validated case definitions, but are missing for many of the psychiatric contracts while psychiatric illness is frequently undetermined and often wrong $(19,20)$. There are patients who suffer from uncontrolled epilepsy, and they may also suffer from excessive or anxiety related to fear of adding to national seizures, loss of work or driving ability, socio-economic depressive status or other psycho protective effects of drugs. The difference between the 
к emotional responses and the predisposition to depressive disorders, being relevant by psychiatric diseases must appear due to the isolation of the biological mechanisms that can underlie both diseases (epilepsy, respectively psychiatric comorbidity) (20).

Cognitive disorders were basic features for unipolar depression, further forming an important criterion for diagnosis (21). This cognitive fear takes on many forms such as reminiscence, lower concentration, difficulties in identifying emotions, prejudices, and a vague reminder of autobiography (22).

\section{DEPRESSION AND EPILEPSY}

Depression is considered the most common psychiatric disorder in people with epilepsy with partial seizures of temporal origin or frontal lobe or in patients with seizures that are not sufficiently controlled $(23,24,25,26)$. The most common symptoms include feelings of archegonia, guilt, but also suicidal ideation. These mood changes are short, stereotypical and appear out of context and appear to be associated with other ictal phenomena (20).

There is in vivo evidence to suggest that patients with depression and epilepsy exhibit neurocognitive network dysfunctions beyond what is seen only in epilepsy (27), being expressed by the potential indicated by the network nodes involved in the priming of pathophysiology of depression in epilepsy (2). However, there is also behavioural evidence that has been made with the help of neuroimaging data, suggesting that the cognitive deficiency that can identify network dysfunctions, if any, with cognitive or psychological affections (28). Functional neuroimaging could indicate that several symptoms of depression may be pathologically associated with two cognitive networks of the brain: the autobiographical memory network (AMN) and the cognitive control network (CNN) (Table no. 1). If the two networks intersect then the AMN is activated when the patient is concerned with introspection and thus the $\mathrm{CCN}$ is inhibited (29)

Depressive mood disorders have an active prevalence and are encountered in $23.1 \%$ of people with epilepsy, the hypotheses of its pathology and the associated factors are: bidirectional relationships (30). Previous studies have shown a national bidirectionality between the 2 disorders as incidence networks being reported with a significant increase for depression before and after diagnosing epilepsy (31).

\begin{tabular}{|l|l|l|}
\hline Networks & Characteristics & Reference \\
\hline AMN & $\begin{array}{l}\text { Self-referential processing, episodic memory, renewal and introspection are } \\
\text { accepted. It includes orbitomesial prefrontal cortex (PFC), rostral anterior } \\
\text { cingulate cortex, hippocampus, posterior cingulate, retrosplenial cortex, } \\
\text { precuneus, and parietal regions important for mental imagery. }\end{array}$ & 2,29 \\
\hline CCN & $\begin{array}{l}\text { Working memory processing, attention and flexible switching between } \\
\text { cognitive sets, dorsolateral recruitment of the PFC and the anterior dorsal } \\
\text { cingulate cortex, with auxiliary nodes in the mesial temporal lobe and } \\
\text { intraparietal sulcus are activated. }\end{array}$ & 2,30 \\
\hline
\end{tabular}

\begin{tabular}{|l|l|l|}
\hline Networks & Characteristics & Reference \\
\hline AMN & $\begin{array}{l}\text { Self-referential processing, episodic memory, renewal and introspection are } \\
\text { accepted. It includes orbitomesial prefrontal cortex (PFC), rostral anterior } \\
\text { cingulate cortex, hippocampus, posterior cingulate, retrosplenial cortex, } \\
\text { precuneus, and parietal regions important for mental imagery. }\end{array}$ & 2,29 \\
\hline
\end{tabular}


Bulletin of Integrative Psychiatry $\bigcirc$ New Series $\bigcirc$ December 2019 ○ Year XXV ONo. 4 (83)/33

\begin{tabular}{|l|l|l|}
\hline \multirow{2}{*}{ CCN } & $\begin{array}{l}\text { Working memory processing, attention and flexible switching between } \\
\text { cognitive sets, dorsolateral recruitment of the PFC and the anterior dorsal } \\
\text { cingulate cortex, with auxiliary nodes in the mesial temporal lobe and } \\
\text { intraparietal sulcus are activated. }\end{array}$ & 2,30 \\
\hline
\end{tabular}

Table I. Characteristics of the two cognitive networks of the brain

\section{DEPRESSION PRE-SEIZURE, SEIZURE AND POSTICTAL}

Depress preictal or prodromal symptoms include depressed mood related panic. It may take from several hours to several days (20). Named and ictal rectal depression is a rare condition with simple expression which is presented by a single partial seizure with episodic dysphoria. Even so, the true prevalence of ictal depression is not yet stable in other studies. These are followed by a change in consciousness as the stroke evolves from a partial evolution to a complex one (20).

Interictal most frequent presentation of depression disorders in patients with epilepsy, being considered as a primary psychiatric disorder (20). Symptoms of depression and its episodes are presented as dysmorphic mood prodromes, can be prolonged on a semi-long term from one day to 3 days before the onset of a seizure, especially by 24 hours . In children, these symptoms are presented by irritability, poor tolerance to frustration and aggressive behavior. (32). Kanner and his colleagues investigated 100 patients with refractory epilepsy, the prevalence rate and clinical features of PS and postictal for three months. The results showed that 43 of the patients regularly experienced a median of about 5 postictal symptoms with an average duration of 24 hours, 25 patients with a history of mood disorders, respectively 11 with anxiety disorders. Postictal depression is for a limited time, with treatment focusing on improved seizure control (33).
Studies published in epilepsy have also listed the following pathogenic mechanisms shared by depression: abnormal activation of the central nervous system (CNS), which has multi neurotransmitters, especially serotonin, neropinefrine, dopamine, GABA and glutamate. There are also structural changes, presenting as atrophic of the temporal and structures with those of the frontal lobe (identified by MRI resolution and volumetric measurement) being found in the amygdaly, hippocampus, entorhinal cortex, temporal, prefrontal and orbital and mesial-frontal lateral neocortex, respectively -a smaller extent in the thalami nuclei and basal ganglia; functional anomalies being identified by positron emission, sion tomography (PET) and single computed tomographic photon emission (SPECT), but also in the frontal lobes, being the basis of the 5HT bond reduction in mesial structures, raphe nuclei, talamus and cingulated girus. In addition, other mechanisms with abnormal functions were found in the hypothalamus-pituitaryadrenal axis anxiety $(20,34)$. Anxiety is also associated with nervousness fear, apprehension and concern, in terms of frequency is the second comorbidity psych IATROS in epileptic patients (15-25\%) (23, $24,26,35,36)$. There it demonstrated that biological variability plays a vague create stress in patients experiencing epileptic seizures, so some researchers have pointed out that's concentration on psycho social predictors variables is leading to anxiety (37, 38). The various forms of anxiety disorders are: generalized anxiety disorder, panic, phobia, obsessive compulsive disorder and post-traumatic disorder. They can 

interactively present the same clinical symptoms autonomous such as tachycardia manifestations as those in the general diaphysis and dyspnea.

population, with neurological factors (TLE) associated with ictal fear (39).

In patients who could be treated with epileptic surgery, this treatment may increase the frequency of anxiety disorders, in some patients it is an onset disorder (40). In pediatric patients, the link between epilepsy and anxiety is harder to observe, the anxiety rate was found to be $16 \%$ in studies conducted by (41), whereas in patients over the age. There are also cross-sectional studies that have shown that the use of primidone, the presence of depression, and a cryptogenic or etiological post-trauma were important predictors for the development of anxiety symptoms (42). Following the research conducted so far, it has been found that there are no specific screening tools for identifying anxiety in patients suffering from epilepsy, but there are some tools that are commonly used in adult patients: the State-Trait Anxiety Scale (STAI). Goldberg depression and anxiety scale, hospital anxiety and depression scale (HADS), Beck's anxiety inventory (BAI), Hamilton anxiety assessment scale (HAM-A or HARS), symptom checklist (SCL-90 -R) and GAD-7 (43).

\section{INTERICTAL ANXIETY AND PHYSIOPATHOLOGY}

Interictal panic consists of episodes lasting between 5 and 20 minutes that can last up to several hours. Feelings of apprehension or anxiety disorder generals (GAD), obsessive compulsive disorder (OCD), post traumatic stress disorder (PTSD), panic disorder (PD) is very intense and is associate with a variety of

In addition, in some patients, the intensity of fear may be so wide that they cannot figure out what is going on around them, without confusion or loss of consciousness, as seen in complex partial seizures. Some patients could also develop agoraphobia due to the fear of experiencing a panic attack in the public (24). In a study on anxiety in patients suffering from epilepsy, a 43-fold increase in agoraphobic anxiety was reported in people without epilepsy, it was considered that this phobia occurs in these patients because of fear of having congestion outside housing (44, 45).

Anxiety disorders are prevalent in life, being evaluated in $22.8 \%$ of the people suffering from epilepsy, having psychotic hypotheses and associated factors such as: tonsil atrophy, GABA-allergic mechanisms, stigma, respectively the impossibility of confiscation (20). There are also studies performed on patients between the ages of 7 and 18 years, respectively the elderly who are experiencing anxiety and depression (Table no. II). In the adult people with epilepsy certain instruments are used to identify if they suffer from anxiety: the state anxiety scale -state (STAI); Goldberg depression and anxiety scale; Hospital Anxiety and Depression Scale (HADS), Beck's Anxiety Inventory (BAI), Hamilton Anxiety Assessment Scale (HAMAA or HARS), A Checklist of Symptoms SCL-90-R and GAD-7 (43).

\begin{tabular}{|l|l|l|}
\hline $\begin{array}{l}\text { Populations with } \\
\text { epilepsy }\end{array}$ & Remarks & Reference \\
\hline $\begin{array}{l}\text { Children and } \\
\text { adolescents }\end{array}$ & $\begin{array}{l}\text { In a study of 44 patients aged between 7 and 18 years, it was } \\
\text { found that there is an anxiety rate of about 26\%. }\end{array}$ & 41 \\
\hline
\end{tabular}


Bulletin of Integrative Psychiatry $\bigcirc$ New Series $\bigcirc$ December $2019 \bigcirc$ Year XXV ONo. 4 (83)/35

\begin{tabular}{|c|c|c|}
\hline & $\begin{array}{l}\text { Another study was conducted on } 35 \text { children and adolescents } \\
\text { between the ages of } 9 \text { and } 18 \text { years. In the group of patients } \\
\text { between the ages of } 9-11 \text { years, there was a significantly higher } \\
\text { trait anxiety, respectively, in the patients between the ages of } 12 \\
\text { and } 18 \text { years, there was a higher anxiety and condition anxiety, } \\
\text { these results were compared with the healthy population. }\end{array}$ & 46 \\
\hline Elderly & $\begin{array}{l}\text { Anxiety disorders in the elderly with epilepsy are difficult to } \\
\text { determine, as little data is known. Many medical conditions are } \\
\text { accompanied by somatic symptoms of anxiety, Certain } \\
\text { medicines used by them such as: steroids, thyroid hormones, } \\
\text { anticholinergic drugs and some antidepressants, can cause } \\
\text { matrix and mental symptoms of anxiety. }\end{array}$ & 36 \\
\hline
\end{tabular}

Table II. Studies performed on populations of patients with epilepsy on anxiety disorders

PREICTAL, ICTAL AND POSTICTAL ANXIETY

Preictal anxiety is defined by the previous symptoms of onset of a crisis, being performed in a period of time from several hours to several days. These premorbid symptoms are stereotypic, helping to predict seizure, anxiety increasing in the days before seizure in patients with partial or complex seizures $(32,46,47)$.

Fear or ictal panic is considered the most common ictal symptom of anxiety of a simple or initial partial seizure of a complex partial seizure and is usually originated in the mesial temporal lobe, but may also exist in the mesial frontal origin. At simple partial seizures these things cannot be observed in certain graphs recorded by the EEG, in relation to the scalp angle the electrodes positioned at the midline and over the suprasilvian regions cannot allow for epileptiform discharges or ictal patterns. The needle ELAS way, scalp recordings are unlikely is to detect epileptiform activity from a simple partial seizure of temporal lobe origin messianic especially those in which the epileptogenic tonsil. At the same time, interictal recordings may fail to recode any interictal discharge, using antero-temporal electrode bases. Sphenoidal electrodes placed under the fluorescence orientation (48) should be used. Epileptic people with ictal panic can sometimes cause interictal panic attacks (36). Postictal anxiety may have relatively common symptoms in patients with partial refractory epilepsy. In a study published in 2004, this comorbidity was studied in 100 PWE patients and it was shown that these symptoms appeared after more than $50 \%$ of the seizures and lasted approximately 24 hours, 32 patients had generalized anxiety, 10 patients reported symptoms of compulsions, and 29 people had postictal symptoms with aspects of agoraphobia (33).

\section{PSYCHOSIS}

Psychotic disorders can be presented as schizophreniform disorders, not being distinguished from those of patients without epilepsy. However, patients with epilepsy and psychosis (POE) have certain characteristics that distinguish them from people who do not have epilepsy. In several studies conducted by Kanner and his collaborators in 1996 and 2004 respectively, the common results included the following: a delay between the onset of psychiatric symptoms and the time of the last seizures, relatively short duration, grouping of symptoms into delusional psychosis similar to vectile, increasing the frequency of generalized seizures secondary to the onset of postictal psychosis (PIP), the 


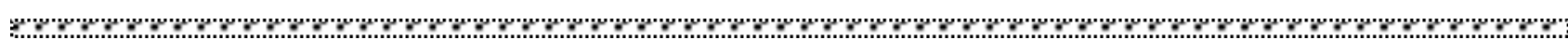

beginning of the PIP after they were confiscated for an average period of more than 10 years and a rapid response to low dose neuroleptic drugs or nezodiazepines (33, 48). In another 8-year study on patients with epilepsy and PIP, the death of 4 out of 14 patients was found, and in 3 patients, chronic psychosis developed (8).

In most cases, insomnia is an initially present symptom in people presenting with recurrent psychotic episodes. These have important localization implications, suggesting the presence of independent bilateral ictal foci. Ictal symptoms and episodes are taken into account each time in the divergence of PIP and POE diagnosis as a whole. Most of the times it is due to the epileptic non-convulsive states, but also the confirmation of the EEG analyzes are essential as a safe psychotic process, such as the catatonic states (lack of reaction) and the mannerisms (automatisms) (8).

\section{PERSONALITY DISORDERS}

The relationship between epilepsy and personality disorders (PD) is often not considered a scientific goal. People with epilepsy are often seen as unstable, introverted and anxious and avoided because of uncertainty. These characteristics correspond to the traits of the C PD cluster (35). In some patients with epilepsy, PD features were detected using a VKP questionnaire (49). These people may suffer from serious emotionality, very ethical as well as spiritual behavior (50), the tendency to be orderly, very detailed, persistent in communication and actions (51), respectively a labile condition with suggestive fathers and immaturity (52). These disorders are estimated to occur in about $4-38 \%$ of people with epilepsy, taking into account the study of heterogeneity (53).

\section{THE TEMPORAL LOBE}

The bidirectional relationship comes from a study of patients with corneal epilepsy of the temporal lobe (TLE), which reveals the progression of psychiatric comorbidity before and after the early control of the offering psychiatric history (54). Personality constructions are considered interactive in nature and are associated with temporal lobe epilepsy. However, there are still no criteria to be elaborated for this to be possible under conditions, and this condition remains controversial (8).

\section{OTHER ATYPICAL SYNDROMES}

Children suffering from epilepsy in combination with attention deficit hyperactivity disorder (ADHD) were asked to perform the same tests as children with only one of the two conditions, so memory types could be analyzed. There were no significant interactions between these conditions, which leads us to think that the three types of patients (children with epilepsy, children with ADHD and children with epilepsy and ADHD) are not significantly different (55).

\section{SUICIDE}

The suicide instinct is the result of one of the comorbid conditions previously presented in relation to epilepsy. In 2017, a study was conducted on the mortality of these patients compared to the global population. The mortality rate (SMR) by suicide in patients with epilepsy was higher (56) than the general population.

From the point of view of suicide attempts, respectively the completed suicide, there are significant differences in the percentage between the population with epilepsy (5$14,3 \%)$ and the general population (1-4,6\%). This standardized mortality ratio of patients with epilepsy appears in those who are institutionalized $(4.6 \%)$ and those with epilepsy in the temporal lobe $(6.6 \%)$, during 


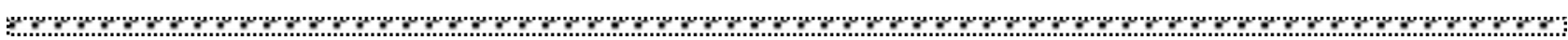

this time it was lower in epileptic persons with new onset (2.1) and those evaluated in tertiary care clinics $(2,3 \%)(20)$.

The suicide rate in patients with epilepsy (PWE) is between 9 and 25 times higher in patients who have seizures of the porous lobe compared to the total population. In the study carried out by Kanner in 2014, out of 100 patients diagnosed with epilepsy, in 13 patients the idea of post-stroke suicide was identified, among them, 10 had a history of major depression are bipolar disorders. Of the 43 patients with postictal depression, 27 of them had concomitant anxiety and 7 reported postictal psychotic symptoms (20).

The putative association between using AED and thinking about a suicide, or complete suicide, is significantly justified. In 2008, the Drug Administration also issued a campaign linking AEDs to suicide. This warning was justified by further analysis of survival and cursing studies conducted on observational cohorts with retrospectives in people with epilepsy, and suggests that the chances of suicidal behavior were 1.84 times higher, as the hazard reports ranged from 1.42 to 2.41 depending on the specific drug after the initiation of AED (20).

\section{PSYCHOTROPIC DRUGS}

Clinical evidence was obtained through questionnaires on human subjects who have epilepsy with different types of seizures, but also people who do not have this condition and have been cataloged as a control. In most cases, the Neurological Disorders Depression Inventory for Epilepsy (NDDI-E) has found that depression is significantly relative to an increased risk of non-adherence to AED drugs, a sense of hopelessness or loss of interest that leads to decreased motivation (57).
Before administering the first dose of antiepileptic drug (AD), it is important to determine if the seizures are associated with the onset or the stroke of an AED. Studies have shown that there is an increased incidence of seizures in people taking maprotiline, amoxepine, clomipramine or bupropion $(58,59)$.

There were cases of patients who were not diagnosed with epilepsy, but following the administration of antidepressant drugs they had the first seizure that was associated with high plasma serum concentrations, a rapid dose, the presence of other drugs with proconvulsive properties and the presence of central nervous system pathology (CNS), abnormal electroencephalon (EEG) and personal and family history of epilepsy (8).

Expression of the toxicity of AED drugs (in terms of frequency: phenobarbital, primidone, topiramate and levetiracetam AED) can be reported as a psychotic disorder. Antidepressants have been particularly controversial in epilepsy. However, it has been noted that the initial concerns were based on ventricular antidepressants (tricyclines and cyclic tetra-antidepressants) in individuals using high doses (60). There are antidepressants that are associated with an increased risk of seizures in epileptic therapy, such as: bupropion, clomipramine, amoxapine, maproline, but they also tend to be associated with increased risk when used at a high dosage level (61).

Treatment of epilepsy may have psychiatric adverse effects consistent with each generation (Table No. III). of agents at the psychological level (20). These psychiatric adverse effects of AEDs are imported because they can affect the etiology of the person. 


\begin{tabular}{|l|l|l|}
\hline Generations & Observation & Reference \\
\hline \multirow{2}{*}{$\begin{array}{l}\text { The first } \\
\text { generation }\end{array}$} & $\begin{array}{l}\text { Benzodiazepines (nitrazapam, clonazepam and cloazam) are associated } \\
\text { bith fatigue and mental retardation, which may induce mood and } \\
\text { Phenytoin causes mental retardation, in rare cases associated with } \\
\text { depression, anxiety and aggression. } \\
\text { Valproic acid is considered a mood stabilizer, but in high doses it can } \\
\text { have adverse effects on the cognitive. } \\
\text { Arbamazepine is considered a state of mind. }\end{array}$ & 62,63 \\
\hline \multirow{2}{*}{$\begin{array}{l}\text { The second } \\
\text { generation }\end{array}$} & $\begin{array}{l}\text { Levetiracetam indicates adverse effects such as behavioral abnormalities, } \\
\text { irritability, aggression and hallucinations. } \\
\text { Topira suggests that there is an irritation between the response and the } \\
\text { dose administered, between drug levels and actual disorders. } \\
\text { It has been proven that a person's mental stress is not related to drugs. }\end{array}$ & 20 \\
\hline
\end{tabular}

Table III. Antiepileptic drugs in both generations

METABOLIC RELEVANCE AND THE "METABOLIC EPILEPSY"

When it comes to the relations between epilepsy and metabolism, there are reports and extensive reviews in the literature on the so-called "Metabolic Epilepsy", which is defined by the Lee group in 2018 as "is a metabolic abnormality which is associated with an increased risk of epilepsy development in affected individuals" (64).

Even more important, as the above authors are describing, it seems that the commune drugs used for treating "normal" epilepsy, are not working in this case, since the mechanistics behind these two are quite different (64). It also seems that diet could be playing an important part in the management of this specific disorder (65).

In fact, in an extensive recent review no less than 14 main mecanistical causes were identified such as: biotinidase deficiency, cerebral folate deficiency, creatine-related disorders, disorders of the urea cycle, folinic acid-responsive seizures, glucose transporter type 1 deficiency syndrome, glutaric aciduria, mitochondrial disorders, molybdenum cofactor deficiency, non-ketotic hyperglycaemia, peroxisomal-related disorders, pyridoxine-dependent epilepsy/ pyridox(am)ine-5'-phosphate oxidase-related deficiency and succinic semialdehyde dehydrogenase (SSADH) Deficiency (64).

In addition, in a related topic there seems to be some related metabolic causes for the epileptic encephalopathy, as described for example by the Yu et al. group in 2013 (66) which are focusing their efforts in describing "defective molecule or mechanism and categorized as small molecule disorders (involving amino and organic acids, fatty acids, neurotransmitters, urea cycle, vitamers and cofactors, and mitochondria) and large molecule disorders (including lysosomal storage disorders, peroxisomal disorders, glycosylation disorders, and leukodystrophies)" in this context (66).

Even more recent papers are also focusing on a so-called diagnostic algorithm for the treatable conditions, for the metabolic manifestations and their evaluation in epilepsy (67). 


\section{CONCLUSIONS}

Psychiatric comorbidity in epilepsy are mainly represented by depression, anxiety, psychosis, personality disorders that sometimes lead to panic, phobia, inattention, irritability, respectively to the thought of suicide and even suicide.

\section{ACKNOWLEDGEMENTS AND DISCLOSURES}

The authors declare that they have no potential conflicts of interest to disclose

\section{REFERENCES}

1. Stafstrom, CE, Carmant, L. Seizures and epilepsy: an overview for neuroscientists. Cold Spring Harbor perspectives in medicine 2015; 5 (6), a022426;

2. Rayner, G. The contribution of cognitive networks to depression in epilepsy. Epilepsy Currents 2017,17 (2), pp. 7883.

3. Pillay, N., Archer, JS, Badawy, RA, et al. Networks underlying paroxysmal fast activity and slow spike and wave in Lennox-Gastaut syndrome. Neurology 2013, 81 (7), pp. 665-673;

4. Liao, W., Zhang, Z., Pan, Z., et al. Default mode network abnormalities in mesial temporal lobe epilepsy: a study combining fMRI and DTI. Human brain mapping 2011; 32 (6), pp. 883-895;

5. Fisher, RS, van Emde Boas, W., Blume, et al. Epileptic seizures and epilepsy: Definitions proposed by the International League Against Epilepsy (ILAE) and the International Bureau for Epilepsy (IBE). Epilepsy 2005; 46, pp. 470-472.

6. Berg, AT, Berkovic, SF, Brodie, MJ et al. Revised terminology and concepts for the organization of seizures and epilepsies: report of the ILAE Commission on Classification and Terminology, 2005-2009. Epilepsy. $2010 ; 51$ (4), pp. 676-685.

7. Hermann, BP, Whitman, S., Hughes, JR, et al. Multietiological determinants of psychopathology and social competence in children with epilepsy. Epilepsy research 1988; 2 (1), pp. 51-60.

8. LaFrance Jr, W. Curt, Andres M. Kanner, and Bruce Hermann. "Psychiatric comorbidities in epilepsy." International review of neurobiology 2008; 83: pp. 347-383.

9. Wittchen, H.-U . Critical Issues in the Evaluation of Comorbidity of Psychiatric Disorders. British Journal of Psychiatry 1996; 168 (S30), pp. 9-16.

10. Goldstein, MA, Harden, CL. Epilepsy and anxiety. Epilepsy \& Behavior 2000; 1 (4), pp. 228-234.

11. Rai, D., Kerr, MP, McManus, S., et al. Epilepsy and psychiatric comorbidity: a nationally representative populationbased study. Epilepsy 2012; 53 (6), pp. 1095-1103.

12. Gaitatzis, A., Trimble, MR, Sander, JW. The psychiatric comorbidity of epilepsy. Acta Neurologica Scandinavica 2004; 110 (4), pp. 207-220.

13. Ottman, R., Lipton, RB, Ettinger, AB, et al. Comorbidities of epilepsy: results from the Epilepsy Comorbidities and Health (EPIC) survey. Epilepsy 2011; 52 (2), pp. 308-315.

14. Tellez-Zenteno, JF, Patten, SB, Jetté, N., et al. Psychiatric comorbidity in epilepsy: a population-based analysis. Epilepsy 2007; 48 (12), pp. 2336-2344.

15. Gaitatzis, A., Carroll, K., Majeed, A., Sander, JW. The epidemiology of comorbidity of epilepsy in the general population. Epilepsy 2004; 45 (12), pp. 1613-1622.

16. Prince, M., Patel, V., Saxena, S., Majet al. No health without mental health. The lancet 2007; 370 (9590), pp. 859 877.

17. Christensen, J., Vestergaard, M., Mortensen, PB, et al. Epilepsy and suicide risk: a population-based case - control study. The Lancet Neurology 2007; 6 (8), pp. 693-698.

18. Thapar, A., Kerr, M., \& Harold, G. Stress, anxiety, depression, and epilepsy: investigating the relationship between psychological factors and seizures. Epilepsy \& Behavior 2009; 14 (1), pp. 134-140.

19. Fiest, Kirsten M., et al. "Systematic review and assessment of validated case definitions for depression in administrative data." BMC psychiatry 2014; 14.1: pp. 289.

20. Josephson, Colin B., Nathalie Jetté. "Psychiatric comorbidities in epilepsy." International review of psychiatry 2017; 29.5 (2017): pp. 409-424.

21. American Psychiatric Association. Diagnostic and statistical manual of mental disorders (DSM-5). American Psychiatric Pub. 2013.

22. Monaco, F. Mood disturbances, psychoses and epilepsy. Epilepsy 1999; 40, pp. 10.

23. Edeh, J., Toone, B .. Relationship between interictal psychopathology and the type of epilepsy. Results of a general practice survey. British Journal of Psychiatry 1987; 151, pp. 95-101.

24. Jacoby, A., Baker, GA, Steen, N., et al. The clinical course of epilepsy and its psychosocial correlates: Findings from a UK Community study. Epilepsy 1996; 37, pp. 148-161.

25. Mendez, MF, Cummings, JL, Benson, DF. Depression in epilepsy. Significance and phenomenology. Archives of Neurology 1986; 43, pp. $766-770$. 
(2)

26. O'Donoghue, MF, Goodridge, DM, Redhead, K., et al. Assessing the psychosocial consequences of epilepsy: A community-based study. Br. J. Gen. Pract 1999; 49, pp. 211-214.

27. Salzberg, M., Taher, T., Davie, M., et al. Depression in temporal lobe epilepsy surgery patients: an FDG-PET study. Epilepsy 2006; 47 (12), pp. 2125-2130.

28. Wilson, SJ, Baxendale, S .. The new approach to classification: rethinking cognition and behavior in epilepsy. Epilepsy \& Behavior 2014; 41, pp. 307-310.

29. Spreng, RN, Grady, CL. Patterns of brain activity supporting autobiographical memory, prospecting, and theory of mind, and their relationship to the default network mode. Journal of cognitive neuroscience 2010; 22 (6), pp. 11121123 .

30. Corbetta, M., Shulman, GL. Goal-directed control and stimulus-driven attention in the brain. Nature reviews neuroscience 2002; 3 (3), pp. 201;

31. Hesdorffer, DC, Ishihara, L., Mynepalli, L., et al. Epilepsy, suicidality, and psychiatric disorders: A bidirectional association. Annals of Neurology 2012; 72, pp. 184-191.

32. Blanchet, P., Frommer, GP. Mood change preceding epileptic seizures. The Journal of Nervous and Mental Disease 1986; 174, pp. 471-476.

33. Kanner AM, Soto A, Gross-Kanner H. Prevalence and clinical characteristics of postictal psychiatric symptoms in partial epilepsy. Neurology 2004; 62: pp. 708-713.

34. Kanner, AM. Depression in epilepsy: A neurobiological perspective. Epilepsy Currents 2005; 5, pp. 21-27.

35. Jones, JE, Hermann, BP, Barry, JJ, et al. Rates and risk factors for suicide, suicidal ideation, and suicide attempts in chronic epilepsy. Epilepsy \& Behavior 2003; 4 (Suppl 3), S31 - S38.

36. Vazquez, B., and Devinsky, O., Epilepsy and anxiety. Epilepsy Behav 2003; 4, S20 - S25.

37. Robertson, MM, Trimble, MR, Townsend, HRA. Phenomenology of depression in epilepsy. Epilepsy 1987; 28 (4), pp. 364-372.

38. Hermann, BP, Whitman, S., Wyler, AR, et al. Psychosocial predictors of psychopathology in epilepsy. The British Journal of Psychiatry 1990; 156 (1), pp. 98-105.

39. Hermann BP, Dikmen S, Schwartz MS, et al. Interictal psychopathology in patients with ictal fear: a quantitative investigation. Neurology 1982; 32: pp. 7-11.

40. Malmgren, K., Sullivan, M., Ekstedt, G., et al. Health-related quality of life after epilepsy surgery: a Swedish multicenter study. Epilepsy 1997; 38 (7), pp. 830-838.

41. Ettinger, AB, Weisbrot, DM, Nolan, EE, et al. Symptoms of depression and anxiety in pediatric epilepsy patients. Epilepsy 1998; 39 (6), pp. 595-599.

42. López-Gómez, M., Espinola, M., Ramirez-Bermudez, J., et al. Clinical presentation of anxiety among patients with epilepsy. Neuropsychiatric disease and treatment 2008; 4 (6), pp. 1235.

43. Kimiskidis, VK, Valeta, T. Epilepsy and anxiety: epidemiology, classification, aetiology, and treatment. Epileptic disorders 2012; 14 (3), pp. 248-256.

44. Beyenburg, S., Mitchell, AJ, Schmidt, D., et al. Anxiety in patients with epilepsy: systematic review and suggestions for clinical management. Epilepsy \& Behavior 2005; 7 (2), pp. 161-171.

45. Gandy, M., Sharpe, L., Perry, KN, et al. Rates of DSM-IV mood, anxiety disorders, and suicidality in Australian adult epilepsy outpatients: a comparison of well-controlled versus refractory epilepsy. Epilepsy \& Behavior 2013 ; 26 (1), pp. 29-35.

46. Oguz A, Kurul S, Dirik E. Relationship of epilepsy-related factors to anxiety and depression scores in epileptic children. J Child Neurol 2002; 17: 37-40.

47. Hughes J, Devinsky O, Feldmann E, et al. Premonitory symptoms in epilepsy. Seizure 1993; 2: 201-3.

48. Kanner, AM, Jones, JC When do sphenoidal electrodes yield additional data to that obtained with antero-temporal electrodes? Electroencephalogr. Gusset. Neurophysiol 1997; 102, pp. 12-19.

49. Swinkels, WAM, Duijsens, IJ, Spinhoven, PH, 2003. Personality disorder traits in patients with epilepsy. Seizure 2003; 12 (8), pp. 587-594.

50. Geschwind, N. Behnavioral Change in Temporal Lobe Epilepsy. Archives of neurology 1997; 34 (8), pp. $453-453$.

51. Blumer, D. Personallty disorders in epilepsy. Neuropsychiatry of personality disorders 1995; pp. 230-263.

52. Schmitz, B., Sander, T. (Eds.). Juvenile myoclonic epilepsy: the Janz syndrome. Routledge 2000.

53. Swinkels, WA, Kuyk, J., van Dyck, R., et al. Psychiatric comorbidity in epilepsy. Epilepsy \& Behavior 2005; 7, pp. 37-50.

54. Jones, JE, Bell, B., Fine, J., et al. A controlled prospective investigation of psychiatric comorbidity in temporal lobe epilepsy. Epilepsy 2007; 48, pp. 2357-2360.

55. Gilliam, F., Kanner, AM Treatment of depressive disorders in epilepsy patients. Epilepsy \& Behavior 2002; 3, pp. $2-9$.

56. Kanner AM, Andres M., Andrey M., et al. "Biomarkers of epileptogenesis: psychiatric comorbidities (?)." Neurotherapeutics 2014; 11.2: 358-372.

57. Ettinger, AB, Good, MB, Manjunath, R. et al. The relationship of depression to antiepileptic drug adherence and quality of life in epilepsy. Epilepsy \& Behavior 2014, 36, pp. 138-143.

58. McConnell, H., Duncan, D. Treatment of psychiatric comorbidity in epilepsy. In " Psychiatric Comorbidity in Epilepsy " (H. McConnell, and P. Snyder, Eds.). American Psychiatric Press, Washington 1998; pp. $245-361$. 
59. Swinkels, J., Jonghe, F. Safety of antidepressants. Int. Gusset. Psychopharmacol 1995; 9, pp. 19-25.

60. Jabbari, B., Bryan, GE, Marsh, EE, et al. Incidence of seizures with tricyclic and tetracyclic antidepressants. Archives of Neurology 1985; 42, pp. 480-481.

61 Kanner, AM Most antidepressant drugs are safe for patients with epilepsy at therapeutic doses: A review of the evidence. Epilepsy \& Behavior 2016; 61, pp. 282-286.

62. Kwan, P., Brodie, MJ Neuropsychological effects of epilepsy and antiepileptic drugs. Lancet 2001; 357, pp. 216222.

63. Mula, M., Sander, JW Negative effects of antiepileptic drugs on mood in patients with epilepsy. Drug Safety 2007; 30, pp. $555-567$.

64. Lin Lin Lee V, Kar Meng Choo B, Chung YS, P Kundap U, Kumari Y, Shaikh MF. Treatment, Therapy and Management of Metabolic Epilepsy: A Systematic Review. Int J Mol Sci 2018; 19, pp. 871.

65. Pong A.W., Geary B.R., Engelstad K.M., Natarajan A., Yang H., De Vivo D.C. Glucose transporter type i deficiency syndrome: Epilepsy phenotypes and outcomes. Epilepsia 2012, 53, pp. 1503-1510

66. Joe Yuezhou Yu and Phillip L. Pearl. Metabolic Causes of Epileptic Encephalopathy. Epilepsy Research and Treatment 2013, pp. 20.

67. van Karnebeek CDM, Sayson B, Lee JJY, Tseng LA, Blau N, Horvath GA and Ferreira CR. Metabolic Evaluation of Epilepsy: A Diagnostic Algorithm With Focus on Treatable Conditions. Front. Neurol 2018, 9, pp. 1016.

\section{Correspondence:}

Alin Ciobîcă,

Researcher, Department of Research, Faculty of Biology, Alexandru Ioan Cuza University, B dul Carol I, no 11, Iasi, Romania Academy of Romanian Scientists, Splaiul Independentei nr. 54, sector 5, 050094 Bucuresti, Romania,Center of Biomedical Research, Romanian Academy, Iasi, B-dul Carol I, no 8, Romania, alinciobica@uaic.ro

Submission: 12 sep 2019

Acceptance: 17 nov 2019 